Pacific Journal of Mathematics

DYNAMICAL SYSTEMS OF CHARACTERISTIC 


\title{
DYNAMICAL SYSTEMS OF CHARACTERISTIC 0
}

\author{
RONALD A. KNIGHT
}

The purpose of this paper is to characterize planar dynamical systems satisfying certain stability criterion. These flows are called dynamical systems of characteristic 0 . Basically the set $S$ of critical points of such a flow is shown to be in one of three categories: $S=\varnothing ; S$ consists of at most two Poincaré centers; or $S=R^{2}$.

1. Introduction. In $\S 2$ we give the basic concepts used throughout the paper. In $\S 3$ we give examples of flows of characteristic $0^{+}$ and $0^{-}$that are not of characteristic 0 . We also give examples of flows of characteristic 0 which are not of characteristic $0^{+}, 0^{-}$, or $0^{ \pm}$. Further, by Examples 2 and 3 we show that the set $S$ of critical points in Theorem 4.8 may actually consist of one or two local Poincaré centers. In $\S 4$ we give necessary and sufficient conditions for a flow $\left(R^{2}, \pi\right)$ to have characteristic 0 .

2. Definitions, notations, and basic theorems. We shall denote the real numbers, nonnegative real numbers, nonpositive real numbers, and Euclidean plane by $R, R^{+}, R^{-}$, and $R^{2}$, respectively. We shall use $R^{2 *}$ to designate the one point compactification of $R^{2}$.

A pair $(X, \pi)$ consisting of a topological space $X$ and a continuous mapping $\pi: X \times R \rightarrow X$ from the product space $X \times R$ into $X$ is called a dynamical system or (continuous) flow whenever the following conditions are satisfied.

1. Identity axiom: $\pi(x, 0)=x$ for each $x \in X$.

2. Homomorphism axiom: $\pi(\pi(x, t), s)=\pi(x, t+s)$ for each $x \in X$ and $t, s \in R$.

3. Continuity axiom: $\pi$ is continuous on $X \times R$.

In this paper $X$ will always be Hausdorff.

We shall let $\pi(x, t)=x t$ for brevity. For each $x \in X, C(x)=x R$, $C^{+}(x)=x R^{+}$, and $C^{-}(x)=x R^{-}$are called the trajectory (or orbit), positive semi-trajectory, and negative semi-trajectory through $x$, respectively. A point $x \in X$ is called a critical or rest point if $x R=x$. If $x$ is not critical and $x t=x$ for some $t>0$, then $x$ is called periodic. For $M \subset X, M$ is said to be invariant if $C(M)=M$ and positively (negatively) invariant if $C^{+}(M)=M\left(C^{-}(M)=M\right)$.

We shall denote the boundary, interior, and closure of a set $M \subset X$ by $\partial M, M^{0}$, and $\bar{M}$, respectively. For any simple closed curve $C$ in $R^{2}$ we shall denote the bounded and unbounded components of $R^{2}-C$ by int $C$ and ext $C$, respectively. We shall let $\eta(x)$ and $\eta(M)$ denote 
the neighborhood filters of $x \in X$ and $M \subset X$, respectively.

The sets $\overline{C(x)}, \overline{C^{+}(x)}$, and $\overline{C^{-}(x)}$ shall be denoted by $K(x), K^{+}(x)$, and $K^{-}(x)$, respectively. The positive (negative) limit set of $x \in X$ is $L^{+}(x)=\bigcap_{t \in R} K^{+}(x t)\left(L^{-}(x)=\bigcap_{t \in R} K^{-}(x t)\right)$. The limit set of $x \in X$ is $L(x)=L^{+}(x) \cup L^{-}(x)$.

A set $M \subset X$ is called positively (orbitally) stable if for every $U \in \eta(M)$ there exists a $V \in \eta(M)$ such that $V=C^{+}(V) \subset U$. Negative and bilateral stability are defined by replacing $C^{+}(V)$ above by $C^{-}(V)$ and $C(V)$, respectively. One can easily verify that a set $M$ is bilaterally stable if and only if $M$ is both positively and negatively stable. When we write stable we shall mean positively stable. by

For each $x \in X$, the positive (negative) prolongation of $x$ is given

$$
D^{+}(x)=\bigcap_{M \in \eta(x)} \overline{C^{+}(M)} \quad\left(D^{-}(x)=\bigcap_{M \in \eta(x)} \overline{C^{-}(M)}\right) .
$$

The prolongation of $x$ is $D(x)=D^{+}(x) \cup D^{-}(x)$. The positive (negative) prolongational limit set of $x$ is given by

$$
J^{+}(x)=\bigcap_{t \in R} D^{+}(x t) \quad\left(J^{-}(x)=\bigcap_{t \in R} D^{-}(x t)\right) .
$$

The prolongational limit set is $J(x)=J^{+}(x) \cup J^{-}(x)$.

The following theorem which we shall use several times in this paper is due to Ura (see [6] and [11]).

Theorem 2.1. Let $X$ be locally compact and $\partial M$ be compact. Then $M$ is stable (negatively stable) if and only if $D^{+}(M)=M\left(D^{-}(M)=M\right.$.) Furthermore, $M$ is bilaterally stable if and only if $D(M)=M$.

A flow $(X, \pi)$ is called parallelizable if it is isomorphic to a parallel flow; that is, if there is a flow $\left(Y \times R, \pi^{\prime}\right)$ such that $(y, t) s=(y, t+s)$ for each $y \in Y$ and $t, s \in R$ and a homeomorphism $f: X \rightarrow Y \times R$ such that $f(x t)=f(x) t$ for each $x \in X$ and $t \in R$. We shall use the following characterization of a parallelizable flow. For a proof see [3] and [4].

Theorem 2.2. Let $X$ be a locally compact separable metric space. A flow $(X, \pi)$ is parallelizable if and only if for each $x \in X, D^{+}(x)=C^{+}(x)$ $\left(D^{-}(x)=C^{-}(x)\right)$ and there are no rest points or periodic orbits.

A flow $(X, \pi)$ is said to have characteristic $0^{+}\left(0^{-}\right)$if $D^{+}(x)=$ $K^{+}(x)\left(D^{-}(x)=K^{-}(x)\right)$ for each $x \in X$. A flow having both characteristic $0^{+}$and $0^{-}$is said to have characteristic $0^{ \pm}$. A flow $(X, \pi)$ is said to have characteristic 0 if $D(x)=K(x)$ for each $x \in X$. The flow $(X, \pi)$ has characteristic $0^{+}\left(0^{-}\right)$if and only if $J^{+}(x)=L^{+}(x)\left(J^{-}(x)=L^{-}(x)\right)$ 
for each $x \varepsilon X$. The corresponding statement does not hold for flows of characteristic $0\left(J\left(x_{0}\right) \neq L\left(x_{0}\right)\right.$ in Example 2).

The basic properties of dynamical systems used in succeeding sections are contained in [5], [6], and [7].

3. Flows of characteristic 0 in $R^{2}$. The characteristic $0^{+}$and $0^{-}$concepts were introduced by Ahmad in [1]. He classified such systems on the plane in terms of their critical points. In [8] necessary and sufficient conditions are given for a flow $\left(R^{2}, \pi\right)$ to have characteristic $0^{+}$or $0^{-}$in terms of the set $S$ of critical points. Ahmad showed that a flow $\left(R^{2}, \pi\right)$ has characteristic $0^{ \pm}$if and only if $S=\varnothing$ and $\left(R^{2}, \pi\right)$ is parallelizable, $S=R^{2}$, or $S=\left\{s_{0}\right\}$ is a global Poincaré center (that is, all trajectories in $R^{2}-\left\{s_{0}\right\}$ are periodic orbits surrounding $s_{0}$ ).

It seems natural to ask whether there is a connection between flows of characteristic 0 and flows of characteristic $0^{+}, 0^{-}$, or $0^{ \pm}$. Since $D^{+}(x)=K^{+}(x)$ and $D^{-}(x)=K^{-}(x)$ for each $x \in R^{2}$ implies $D(x)=K(x)$, any flow of characteristic $0^{ \pm}$is a flow of characteristic 0. A flow which has characteristic $0^{+}\left(0^{-}\right)$but not characteristic 0 is given below in Example 1. Examples 2 and 3 consist of flows of characteristic 0 that are not of characteristic $0^{+}, 0^{-}$, or $0^{ \pm}$.

Example 1. The system of differential equations

$$
\begin{aligned}
& \dot{x}=-x \\
& \dot{y}=-y
\end{aligned}
$$

defines a flow of characteristic $0^{+}$in which the origin is a proper node. Note, however, that $D((0,0))=R^{2} \neq\{(0,0)\}=K((0,0))$ so that the flow does not have characteristic 0 .

Similarly, the flow defined by $\dot{x}=x$ and $\dot{y}=y$ is of characteristic $0^{-}$but not of characteristic 0 .

EXAMPLE 2. Let a flow be defined by the system

$$
\begin{gathered}
\dot{r}=-r^{2} \sin \theta \\
\dot{\theta}=1
\end{gathered}
$$

for $r \geqq 0$. Figure 1 illustrates the trajectories of the flow.

This flow is of characteristic 0 but not characteristic $0^{+}, 0^{-}$, or $0^{ \pm}$. For let $x_{0}$ be a point on the parabolic boundary of the region consisting of the pole and the periodic orbits surrounding the pole. Then $D\left(x_{0}\right)=D^{+}\left(x_{0}\right)=D^{-}\left(x_{0}\right)=C\left(x_{0}\right)=K\left(x_{0}\right)$ implies that $D^{+}\left(x_{0}\right) \neq$ $K^{+}\left(x_{0}\right)$ and $D^{-}\left(x_{0}\right) \neq K^{-}\left(x_{0}\right)$.

Example 3. The flow defined by the system of differential equations 

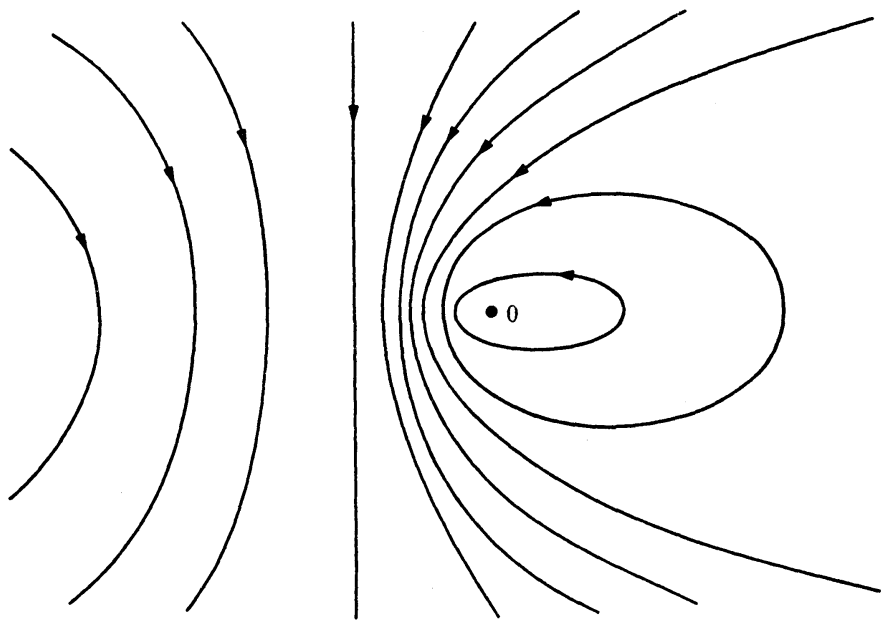

Figure 1

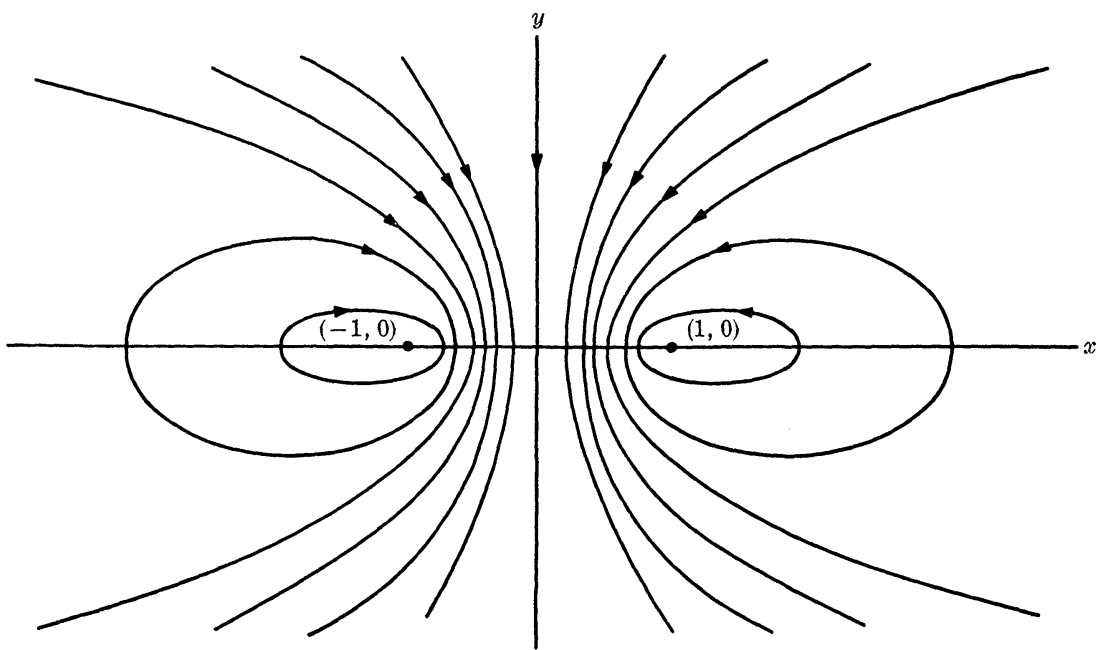

FIGURE 2

$\dot{x}=-x y$

$$
\dot{y}=\left\{\begin{array}{r}
x-1-y^{2} \quad \text { for } x \geqq 0 \\
-x-1-y^{2} \quad \text { for } x<0
\end{array}\right.
$$

is of characteristic 0. After changing system (1) to Cartesian coordinates, system (2) can be obtained by translation and reflection. The phase plane of (2) is illustrated in Figure 2.

4. Characterization of flows having characteristic 0 . The purpose of this section is to give necessary and sufficient conditions for a flow $\left(R^{2}, \pi\right)$ to have characteristic 0 . Unless otherwise specified we shall let $\left(R^{2}, \pi\right)$ be a fixed flow of characteristic 0 and $S$ be the set 
of critical points. We shall first prove a few lemmas.

Lemma 4.1. If $L^{+}(x) \neq \varnothing\left(L^{-}(x) \neq \varnothing\right)$ for some $x \in R^{2}$, then $x$ is either periodic or critical.

Proof. Let $y \in L^{+}(x)$. Then $x \in J^{-}(y)$ since $y \in J^{+}(x)$. Hence, $x \in D(y)=K(y) \subset L^{+}(x)$. Seibert and Tulley have shown in [10] that a point is positively (negatively) Poisson stable if and only if it is either a critical point or a periodic point. The result for $L^{-}(x) \neq \varnothing$ follows similarly.

Lemma 4.2. If $x \in S$ or $x$ is periodic, then $C(x)$ is bilaterally stable.

Proof. The proof follows from Theorem 2.1 since $D(x t)=K(x t)=$ $K(x)=C(x)$ for each $t$ in $R$ implies $D(C(x))=C(x)$.

Notation. For any $s \in S$ we shall henceforth let

$$
N_{s}=\left\{x \in R^{2}: x=s \text { or } x \text { is periodic and } S \cap \operatorname{int} C(x)=\{s\}\right\} .
$$

LEMmA 4.3. If $s_{0}$ is an isolated point of $S$, then $s_{0}$ is a Poincaré center and $N_{s_{0}}$ is an unbounded connected open set. If $N_{s_{0}} \neq R^{2}$, then $\partial N_{s_{0}}$ is a single trajectory and $N_{s_{0}}$ is a simply connected component of $R^{2}-\partial N_{s_{0}}$.

Proof. Let $C$ be a simple closed curve with $S \cap \operatorname{int} C=\left\{s_{0}\right\}$. By virtue of Lemma 4.2 there exists a $V \in \eta\left(s_{0}\right)$ such that $C(V) \subset \operatorname{int} C$. Since $L^{+}(x) \neq \varnothing$ for each $x \in V, V-\left\{s_{0}\right\}$ consists of periodic points. If $x \in V-\left\{s_{0}\right\}$ then $\varnothing \neq S \cap \operatorname{int} C(x) \subset S \cap$ int $C=\left\{s_{0}\right\}$. Thus, $V$ consists of $s_{0}$ and periodic orbits surrounding $s_{0}$ implying that $s_{0}$ is a Poincaré center.

Let $x \in N_{s_{0}}-\left\{s_{0}\right\}$ and $y \in($ int $C(x))-\left\{s_{0}\right\}$. Since $L^{+}(y) \neq \varnothing, y$ is periodic. We have $\varnothing \neq S \cap \operatorname{int} C(y) \subset S \cap \operatorname{int} C(x)=\left\{s_{0}\right\}$ so that $y \in N_{s_{0}}$. Hence, int $C(x) \subset N_{s_{0}}$. Furthermore, $N_{s_{0}}$ is connected since $N_{s_{0}}=$ $\bigcup_{x \in N s_{0}} \overline{\text { int } C(x)}$ is the union of connected sets each containing the point $s_{0}$.

If $\partial N_{s_{v}}=\varnothing$ then $N_{s_{0}}=R^{2}$ and $s_{0}$ is a global Poincaré center. Suppose $\partial N_{s_{0}} \neq \varnothing$. Note that $\partial N_{s_{0}}$ is invariant since $N_{s_{0}}$ is invariant. We shall show that in this case $\partial N_{s_{0}}$ contains no critical points or periodic points. First, suppose $s \in \partial N_{s_{0}} \cap S$. There is an open simply connected neighborhood $U$ in $\eta(s)$ such that $s_{0} \notin U$. By Lemma 4.2 there exists a $V_{1} \in \eta(s)$ such that $C\left(V_{1}\right) \subset U$. Let $x \in V_{1} \cap N_{s_{0}}$. Then $C(x) \subset U$. Since $U$ is simply connected, $s_{0} \in \operatorname{int} C(x) \subset U$ which is a 
contradiction. Hence, $S \cap \partial N_{s_{0}}=\varnothing$. Next, suppose there is a periodic point $x$ in $\partial N_{s_{u}}$. Let $S_{0}=S \cap$ int $C(x)$. There is a simply connected neighborhood $U \in \eta(C(x))$ such that $S \cap U=S_{0}$. By Lemma 4.2 there is a $V_{2} \in \eta(C(x))$ such that $C\left(V_{2}\right) \subset U$. For $y \in\left(N_{s_{0}} \cap V_{2}\right)-\left\{s_{0}\right\}$ we have $C(y) \subset V_{2}$. Since $U$ is simply connected, int $C(y) \subset U$. Hence, $s_{0} \in S \cap$ $U=S_{0}$. The sets $S_{0}$ and $S-S_{0}$ are closed, so there are simple closed curves $C_{1}$ and $C_{2}$ contained in int $C(x)$ and ext $C(x)$, respectively, such that $S \cap\left(\operatorname{ext} C_{1}\right) \cap\left(\right.$ int $\left.C_{2}\right)=\varnothing$. By Lemma 4.2 there is a $V_{3} \in \eta(C(x))$ such that $C\left(V_{3}\right) \subset\left(\operatorname{ext} C_{1}\right) \cap\left(\operatorname{int} C_{2}\right)$. Now $N_{s_{0}}$ is connected with $s_{0} \in N_{s_{0}}$ and $C(x) \subset \partial N_{s_{0}}$, so that we can select a point $y$ from $N_{s_{0}} \cap V_{3} \cap \operatorname{int} C(x)$. Thus, $S_{0} \cap \operatorname{int} C(y) \neq \varnothing, S_{0} \subset \operatorname{int} C_{1}$, and $C(y) \subset V_{3} \subset \operatorname{ext} C_{1}$ imply $S_{0} \subset$ $\operatorname{int} C(y)$. Hence, $S_{0}=\left\{s_{0}\right\}$ and $x \in N_{s_{0}}$. Finally, for any point $z \in V_{3} \cap$ ext $C(x), L^{+}(z) \neq \varnothing$ implying $z$ is periodic. Since $C(z) \subset C\left(V_{3}\right) \subset \operatorname{int} C_{2}$ we have $S \cap \operatorname{int} C(z)=S_{0}$. The point $z$ is in $N_{s_{0}}$ and $C(x) \subset \operatorname{int} C(z) \subset N_{s_{0}}^{0}$. This contradicts $x \in \partial N_{s_{0}}$. Therefore, the points of $\partial N_{s_{0}}$ are neither periodic nor critical.

By virtue of Lemma 4.1 and the fact that $\partial N_{s_{0}}$ contains no periodic or rest points, $L^{ \pm}(x)=\varnothing$ for each $x \in \partial N_{s_{0}}$. Thus, $\partial N_{s_{0}}$ is not bounded and hence $N_{s_{0}}$ is an unbounded open set.

We now show that $\partial N_{s_{0}}$ is a single trajectory. Let $x$ and $y$ be distinct points of $\partial N_{s_{0}}$. Let $C_{1}$ and $C_{2}$ be simple closed curves such that $x \in \operatorname{int} C_{1}, y \in \operatorname{int} C_{2}$, and $\overline{\operatorname{int} C_{1}} \cap \overline{\operatorname{int} C_{2}}=\varnothing$. For $z$ in $N_{s_{0}} \cap \operatorname{int} C_{1}$ we have $\overline{\operatorname{int} C(z)} \subset N_{s_{0}}$, and so ext $C(z) \in \eta(y)$. Thus, (int $\left.C_{2}\right) \cap$ $(\operatorname{ext} C(z)) \in \eta(y)$ and $\left(\operatorname{int} C_{2}\right) \cap\left(\operatorname{ext}(C(z)) \cap N_{s_{0}} \neq \varnothing\right.$. Let $w \in\left(\operatorname{int} C_{2}\right) \cap$ $(\operatorname{ext} C(z)) \cap N_{s_{0}}$. Then $C(z) \subset \operatorname{int} C(w) \subset N_{s_{0}}$. We have $z \in \operatorname{int} C(w)$ and $x \in \operatorname{ext} C(w)$. Since $x, z \in \operatorname{int} C_{1}$ and $\operatorname{int} C_{1}$ is connected, it follows that $C(w) \cap \operatorname{int} C_{1} \neq \varnothing$. Hence, we can find nets $\left(w_{i}\right)$ and $\left(w_{i} t_{i}\right)$ converging to $y$ and $x$, respectively. In other words, $x \in D(y)=K(y)=C(y)$.

Suppose $N_{s_{0}}$ is not a component of $R^{2}-\partial N_{s_{0}}$. Since $N_{s_{0}}$ is connected, it is a subset of some component $B$. If $N_{s_{0}} \neq B$, then $\partial N_{s_{0}} \cap$ $B \neq \varnothing$ contradicting $B \subset R^{2}-\partial N_{s_{0}}$. Hence, $N_{s_{0}}$ is a component of $R^{2}-\partial N_{s_{0}}$.

Finally, let $R^{2} \neq N_{s_{0}}$. Suppose that $C$ is a simple closed curve lying in $N_{s_{0}}$ with int $C \not \subset N_{s_{0}}$. Then int $C$ connected and $N_{s_{0}} \cap \operatorname{int} C \neq$ int $C$ imply that $\partial N_{s_{0}} \cap \operatorname{int} C \neq \varnothing$. Furthermore, $\partial N_{s_{0}} \cap \operatorname{ext} C \neq \varnothing$ since $\partial N_{s_{0}}$ is unbounded. Thus, $C \cap \partial N_{s_{0}} \neq \varnothing$ contradicting $C \subset N_{s_{\vartheta}}$. Therefore, $N_{s_{0}}$ is simply connected.

Lemma 4.4. If $S_{0}=S \cap \operatorname{int} C\left(x_{0}\right)$ for some periodic point $x_{0}$, then $S_{0}$ consists of exactly one Poincaré center.

Proof. Let $N=\left\{x \in \operatorname{int} C\left(x_{0}\right): x\right.$ is periodic and $\left.S_{0}=S \cap \operatorname{int} C(x)\right\}$ and $D=\bigcap_{x \in N} \overline{\operatorname{int} C(x) \text {. }}$ At least $x_{0} \in N$, so that $D \neq \varnothing$. Also, $D$ is the intersection of closed invariant sets containing $S_{0}$ so that $D$ is a 
closed invariant set and $S_{0} \subset D$. It also follows that $\partial D$ is invariant.

In order to facilitate the argument we show that $V \in \eta(C(y))$ implies $V \cap N \neq \varnothing$ for all $y \in \partial D$. Suppose $V \cap N=\varnothing$ for some $V \in \eta(C(y))$. By Lemma 4.2 there is a connected set $U \in \eta(C(y))$ such that $C(U) \subset V$. For $x \in N, U \cap C(x)=\varnothing$. Since $y \in \operatorname{int} C(x)$ and $U$ is connected we have $U \subset \operatorname{int} C(x)$. The point $x$ was arbitrary, so that $U \subset D$. But this implies $y \in D^{0}$ which contradicts $y \in \partial D$.

Suppose that $D$ is not a singleton. We first show that there exists a point $y \in N$ such that $D=\overline{\operatorname{int} C(y)}$. If $D=\overline{\operatorname{int} C\left(x_{0}\right)}$ then we are done. Assume $D \neq \overline{\operatorname{int} C\left(x_{0}\right)}$ and choose points $x$ in $D$ and $y$ in $\partial D$ such that $x \neq y$. Either $y \in S_{0}$ or $y$ is periodic. Suppose there exists a simple closed curve $C$ such that $x \in \operatorname{ext} C$ and $C(y) \subset \operatorname{int} C$. By Lemma 4.2 there is a $V \in \eta(C(y))$ such that $C(V) \subset \operatorname{int} C$. We have shown that $V \cap N \neq \varnothing$. Let $z \in V \cap N$. Then $C(z) \subset C(V) \subset \operatorname{int} C$. But this implies that $x \in \operatorname{int} C(z) \subset \operatorname{int} C$ contradicting $x \in$ ext $C$. Thus, $y$ is periodic and $x \in \operatorname{int} C(y)$. Since $x$ was an arbitrary point of $D$, we have $D \subset \overline{\operatorname{int} C(y)}$. Furthermore, $C(y) \subset \partial D \subset \operatorname{int} C(z)$ for each $z \in N$ implying $\overline{\operatorname{int} C(y)} \subset \bigcap_{z \in N} \overline{\operatorname{int} C(z)}=D$. Hence, $D=\overline{\operatorname{int} C(y)}$.

Since $S_{0}$ is compact there exists a simple closed curve $C \subset \operatorname{int} C(y)$ with $S_{0} \subset \operatorname{int} C$. By Lemma 4.2 there is a $V \in \eta(C(y))$ such that $C(V) \subset$ ext $C$. Each point $z$ in $V \cap \operatorname{int} C(y)$ is periodic by Lemma 4.1, and so, $S_{0} \cap \operatorname{int} C(z) \neq \varnothing$. Since $C(z) \subset \operatorname{ext} C$, int $C(z) \cap \operatorname{int} C \neq \varnothing$, and int $C$ is connected, we have $S_{0} \subset \operatorname{int} C \subset \operatorname{int} C(z)$ and $z \in N$. Thus, $D \subset \overline{\operatorname{int} C(z)}$ and $C(z) \subset \operatorname{int} C(y)$ imply that $D \subset \operatorname{int} C(y)$ which contradicts $y \in D$. Consequently, $D$ must be a singleton.

Finally, $\varnothing \neq S_{0} \subset D$ implies that $D$ is composed of an isolated critical point. By Lemma 4.3, $S_{0}$ consists of a Poincaré center.

Lemma 4.5. If $S \neq \varnothing$ and $S \neq R^{2}$, then $S$ consists of Poincaré centers.

Proof. Let $S_{0}$ denote the set of Poincaré centers. We can select a point $s$ from $\partial S$ since $S \neq \varnothing$ and $S \neq R^{2}$. For any compact set $U \in \eta(s)$ there exists a $V \in \eta(s)$ such that $C(V) \subset U$ by Lemma 4.2. For any $x \in V \cap\left(R^{2}-S\right), L^{+}(x) \neq \varnothing$ implying that $x$ is periodic. Thus, Lemma 4.4 implies $S_{0} \neq \varnothing$.

Suppose $s \in \partial\left(S-S_{0}\right)$. Since $s$ is bilaterally stable, $\eta(s)$ contains a compact connected simply connected invariant set $V$. Either $V$ contains a regular point or a center. If it contains a regular point $x$, then $x$ must be periodic so that int $C(x)$, and hence $V$, must contain a center. Therefore, we can assume that $V$ contains a center $s_{0}$. Now, for each $x \in N_{s_{0}}-\left\{s_{0}\right\}, s_{0} \in \operatorname{int} C(x)$ and, by Lemma 4.4, $s \in \operatorname{ext} C(x)$. Thus, $V$ must meet $C(x)=\partial \operatorname{int} C(x)$ since it is connected. But this implies $C(x) \subset V$ and hence $N_{s_{0}} \subset V$, contradicting Lemma 
4.3. Therefore, $\partial\left(S-S_{0}\right)=\varnothing$, and so $S=S_{0}$.

Lemma 4.6. If $S \neq \varnothing$ and $S \neq R^{2}$, then $S$ consists of at most two Poincaré centers.

Proof. Suppose $s_{1}, s_{2}$, and $s_{3}$ are distinct points of $S$. We shall show that this supposition leads to a countable collection of mutually disjoint closed sets whose union is $R^{2}$ which is impossible. Unless explicitly stated, the remainder of the proof will be considered relative to the extended dynamical system on $R^{2 *}$. We denote the closure of the trajectory through $x$ in the extended system by $K^{*}(x)$.

Since the sets $N_{s}$ are disjoint and open relative to $R^{2}, A=R^{2}-$ $\bigcup_{s \in S} N_{s}$ is nonempty. For each $x \in A, K^{*}(x)=C(x) \cup\{\infty\}$ is a simple closed curve. Let $M=\left\{x \in A: N_{s_{1}} \subset A_{x}\right.$ and $N_{s_{2}} \cup N_{s_{3}} \subset B_{x}$ where $A_{x}$ and $B_{x}$ are the components of $\left.R^{2 *}-K^{*}(x)\right\}$. By Lemma 4.3, $M \neq \varnothing$ since $\partial N_{s_{1}}-\{\infty\} \subset M$. Note that $\overline{A_{x}}=A_{x} \cup K^{*}(x)$ and let $F_{s_{1}}=\mathrm{U}_{x \in M} \overline{A_{x}}$. Each set $\overline{A_{x}}$ is connected and contains $N_{s_{1}}$, and so $F_{s_{1}}$ is connected.

For any point $p_{1}$ in $\partial F_{s_{1}}-\{\infty\}$ we have $F_{s_{1}}=\overline{A_{p_{1}}}$. For let $p_{1}$ and $q_{1}$ be distinct points in $\partial F_{s_{1}}-\{\infty\}$ and let $C_{1}$ and $C_{2}$ be simple closed curves in $R^{2}$ surrounding $p_{1}$ and $q_{1}$, respectively, such that $\overline{\text { int } C_{1}} \cap \overline{\operatorname{int} C_{2}}=\varnothing$. There exists a point $p$ for which $A_{p} \cap \operatorname{int} C_{1}$, and hence $C(p) \cap \operatorname{int} C_{1}$, are nonempty sets. Since $B_{p} \cap \operatorname{int} C_{2} \in \eta\left(q_{1}\right)$ there exists a point $q$ such that $A_{q} \cap B_{p} \cap$ int $C_{2} \neq \varnothing$; hence, $C(q) \cap \operatorname{int} C_{2} \neq \varnothing$. Now, $A_{q}$ meets $A_{p}$ and $B_{p}$, so that $A_{p} \subset A_{q}$. Thus, $A_{q}$ is a connected set which meets both int $C_{1}$ and ext $C_{1}$ implying that $C(q) \cap \operatorname{int} C_{1} \neq \varnothing$. We can find nets $\left(x_{i}\right)$ and $\left(x_{i} t_{i}\right)$ converging to $q_{1}$ and $p_{1}$, respectively; hence, $p_{1} \in D\left(q_{1}\right)=K\left(q_{1}\right)=C\left(q_{1}\right)$ and $\partial F_{s_{1}}-\{\infty\}=C\left(p_{1}\right)$. Now, $C\left(p_{1}\right) \not \subset N_{s}$ for any $s$ in $S$ since $N_{s} \cap F_{s_{1}} \neq \varnothing$ implies there exists an $x$ in $M$ such that $C\left(p_{1}\right) \subset N_{s} \subset A_{x} \subset F_{s_{1}}^{0}$ contradicting $C\left(p_{1}\right) \subset \partial F_{s_{1}}$. Thus, $C\left(p_{1}\right) \subset A$. Since $F_{s_{1}}$ is an invariant set, either $C\left(p_{1}\right) \subset F_{s_{1}}$ or $C\left(p_{1}\right) \cap F_{s_{1}}=\varnothing$. Suppose $C\left(p_{1}\right) \cap F_{s_{1}}=\varnothing$. Then $F_{s_{1}}-\{\infty\}$ is the connected set $F_{s_{1}}^{0}$, and so it is a component of $R^{2 *}-K^{*}\left(p_{1}\right)=R^{2 *}-\partial F_{s_{1}}$. Also, $N_{s_{2}} \cup$ $N_{s_{3}} \subset \bigcap_{x \in M} B_{x}=R^{2^{*}}-F_{s_{1}}$ which means $p_{1} \in F_{s_{1}}$, contradicting $C\left(p_{1}\right) \cap$ $F_{s_{1}}=\varnothing$. Hence, $F_{s_{1}}=\bar{A}_{p_{1}}$.

Analogously, for $s_{2}$ and $s_{3}$ there exists points $p_{2}$ and $p_{3}$ in $A$ and sets $F_{s_{2}}$ and $F_{s_{3}}$ such that $F_{s_{2}}=\bar{A}_{p_{2}}^{\prime}$ and $F_{s_{3}}=\bar{A}_{p_{3}}^{\prime \prime}$. Note that $F_{s_{1}}=$ $\bar{A}_{p_{1}}$ and $F_{s_{2}} \subset B_{p_{1}}$. If $\partial F_{s_{1}}=K^{*}\left(p_{1}\right)=K^{*}\left(p_{2}\right)=\partial F_{s_{2}}$ then $F_{s_{1}} \cup F_{s_{2}}=R^{2 *}$ which contradicts $s_{3} \notin F_{s_{1}} \cup F_{s_{2}}$. Hence, $F_{s_{1}} \cap F_{s_{2}}=\{\infty\}$. Similarly, $F_{s_{1}} \cap F_{s_{3}}=F_{s_{2}} \cap F_{s_{3}}=\{\infty\}$.

Let $F=\bigcup_{i=1}^{3} F_{s_{i}}$. Obviously, $R^{2 *} \neq F$, and so $R^{2 *}-F \neq \varnothing$. Suppose that $A \cap\left(R^{2 *}-F\right)=\varnothing$. Then $R^{2^{*}}-F$ must consist of periodic and rest points, so that $N_{s} \subset R^{2 *}-F$ for some $s \in S$. Furthermore, $\partial N_{s}-\{\infty\} \subset A$ implies that $\partial N_{s} \subset \partial F=\bigcup_{i=1}^{3} K^{*}\left(p_{i}\right)$. By letting $\partial N_{s}=$ $K^{*}\left(p_{k}\right)$ we have $R^{2 *}=N_{s} \cup F_{s_{k}}$ since $N_{s}$ and $F_{s_{k}}^{0}$ are components of 
$R^{2 *}-K^{*}\left(p_{k}\right)$. But this implies that $s_{i} \in F_{s_{k}}$ for $i \neq k$ which is clearly not possible. Therefore, $A \cap\left(R^{2 *}-F\right) \neq \varnothing$.

For each point $x$ in $A \cap\left(R^{2 *}-F\right)$ one component $R^{2^{*}}-K^{*}(x)$ contains $F$ since $K^{*}(x)$ does not separate any of the sets $N_{s_{1}}, N_{s_{2}}$, and $N_{s_{3}}$ from the other two. Denote the components of $R^{2 *}-K^{*}(x)$ by $G_{x}$ and $H_{x}$ where $F \subset H_{x}$. For any point $y$ in $A \cap\left(R^{2^{*}}-F\right)$, let $M_{y}=\left\{x \in A \cap\left(R^{2 *}-F\right): G_{y} \subset G_{x}\right\}$. Note that $M_{y} \neq \varnothing$ since $y \in M_{y}$. Let $F_{y}^{\prime}=\bigcup_{x \in M_{y}} G_{x}$. By arguing as we did for $F_{s_{1}}$, we can find a point $w$ in $\partial F_{y}^{\prime} \cap A$ such that $F_{y}^{\prime}=\bar{G}_{w}$. For each point $p$ in $A \cap\left(R^{2^{*}}-F\right)$ for which $F_{p}^{\prime}=F_{y}^{\prime}$, select a point $y_{0}$ in $C(w)$ and denote $F_{p}^{\prime}$ by $F_{y_{0}}$. Let $I^{\prime}$ be the index set for all the $F_{y_{0}}$ sets and let $I=I^{\prime} \cup\left\{s_{1}, s_{2}, s_{3}\right\}$.

If $x$ and $z$ are distinct points in $I$, then $F_{x} \cap F_{z}=\{\infty\}$. For suppose $F_{x} \cap F_{z} \neq\{\infty\}$. The sets $F_{x}^{0}$ and $F_{z}^{0}$ are components of $R^{2^{*}}-\partial F_{x}$ and $R^{2^{*}}-\partial F_{z}$, respectively, where $\partial F_{x}$ and $\partial F_{z}$ are simple closed curves each consisting of $\{\infty\}$ and a single trajectory. Thus, either $\partial F_{x}-\{\infty\} \subset F_{z}^{0}, \partial F_{z}-\{\infty\} \subset F_{x}^{0}$, or $F_{x}^{0} \cap F_{z}^{0}=\varnothing$. The first two statements imply that $F_{x}=F_{z}$, and hence $x=z$, contradicting $x \neq z$. The third statement implies that $F_{x} \cup F_{z}=R^{2 *}$ which is impossible. Therefore, $F_{x} \cap F_{z}=\{\infty\}$.

Next, $R^{2 *}=\bigcup_{x \in I} F_{x}$. For let $z$ belong to $R^{2 *}-E$ where $E=$ $\bigcup_{x \in I} F_{x}$. Since $A \subset E$, there is a point $s$ in $S$ such that $z \in N_{s}$. For some point $y$ in $E, K^{*}(y)=\partial N_{s}$. Furthermore, there is a point $x$ in $I$ such that $K^{*}(y)=\partial F_{x}$ since $K^{*}(y) \subset \partial E$. The sets $N_{s}$ and $F_{x}^{0}$ are disjoint components of $R^{2 *}-K^{*}(y)$, and so $R^{2 *}=N_{s} \cup F_{x}$. This implies $F_{x}=E$, and thus $s_{i} \in F_{x}$ for $i=1,2,3$, which is clearly impossible. Hence, $R^{2 *}=E$.

The set $\left\{F_{x}: x \in I\right\}$ is a countable collection of closed sets such that $F_{x} \cap F_{z}=\{\infty\}$ for $x \neq z$. Hence, $\left\{F_{x}-\{\infty\}: x \in I\right\}$ is a countable collection of mutually disjoint sets closed in $R^{2}$ and $R^{2}=\bigcup_{x \in I}\left(F_{x}-\{\infty\}\right)$. This is not possible as we indicated at the outset of our argument. Therefore, $s_{1}, s_{2}$, and $s_{3}$ are not distinct.

Lemma 4.7. Let $S \neq R^{2}$. Then the flow restricted to $R^{2}-\mathbf{U}_{s \in S} N_{s}$ is parallelizable.

Proof. Let $Y=R^{2 *}-\bigcup_{s \in S} N_{s}$. The set $\{\infty\}$ is compact and invariant. According to Theorem 2 p. 151 of [9], $J(x)=\infty$ for each $x$ in $Y-\{\infty\}$. Thus, relative to $Y-\{\infty\}, J(x)=\varnothing$ and $D^{+}(x)=C^{+}(x)$. The result follows by Theorem 2.2.

THEOREM 4.8. A flow $\left(R^{2}, \pi\right)$ has characteristic 0 if and only if one of the following holds.

(1) $S=\varnothing$ and $\left(R^{2}, \pi\right)$ is parallelizable.

(2) $S$ consists of at most two Poincaré centers. For each $s \in S$, 
either $s$ is a global Poincaré center or $N_{\mathrm{s}}$ is unbounded and $\partial N_{\mathrm{s}}$ is a single trajectory. The restriction of the flow to $R^{2}-U_{s \in S} N_{s}$ is parallelizable.

(3) $S=R^{2}$.

Proof. The necessity of the conditions follows from the lemmas. Conversely, Theorem 2.2 shows that condition (1) is sufficient. Similarly, if condition (2) holds, we get $D(x)=K(x)$ for each $x \in R^{2}-$ $\mathrm{U}_{s \in S} N_{s}$. For each $s \in S, N_{s}$ is a component of $R^{2}-\partial N_{s}$ since $\partial N_{s}$ is a single trajectory. Thus, $N_{s}$ is a connected simply connected set. Obviously, $x \in \bar{N}_{s}$ implies $D(x)=K(x)$. Hence, condition (2) is sufficient. Condition (3) is trivially sufficient.

Corollary 4.9. A flow $\left(R^{2}, \pi\right)$ has characteristic 0 if and only if $D(x)=C(x)$ for each $x \in R^{2}$.

REMARK. That there are six basic types of planar flows (up to dynamical isomorphism) having characteristic 0 follows from Theorem 4.8. These are

(1) parallelizable flows,

(2) flows having a global Poincaré center,

(3) flows similar to Example 2,

(4) flows similar to Example 3,

(5) flows similar to Example 3 except that $\partial N_{s}=\partial N_{t}$ where $S=$ $\{s, t\}$, and

(6) flows having only critical points.

Note that only the flows in (1), (2), and (6) have characteristics $0,0^{+}$, $0^{-}$, and $0^{ \pm}$and that the flows in (3), (4), and (5) have only characteristic 0 .

The author is grateful to his $\mathrm{Ph}$. D. research advisor, Professor Shair Ahmad, for suggesting that he characterize flows of characteristic 0 as part of his thesis research. This paper is based on Chapter 3 of the thesis.

\section{REFERENCES}

1. S. Ahmad, Dynamical systems of characteristic $0^{+}$, Pacific J. Math., 32 (1970), 561-574.

2. Strong attraction and classification of certain continuous flows, Math. Systems Th., 5 (1971), 157-163.

3. H. Antosiewicz and J. Dugundji, Parallelizable flows and Liapunov's second method, Ann. of Math., 73 (1961), 543-555.

4. N. Bhatia, Criteria for dispersive flows, Math. Nach., 32 (1960), 89-93.

5. N. Bhatia and O. Hajek, Local semi-dynamical systems, Lecture Notes in Math. No. 90, Springer-Verlag, New York, 1969.

6. Theory of dynamical systems, Parts I and II, Technical Notes BN-599 and BN-606, Univ. of Maryland, 1969. 
7. O. Hajek, Dynamical Systems on the Plane, Academic Press, New York, 1968.

8. R. Knight, Ph. D. Dissertation, Oklahoma State University, 1971.

9. T. Saito, On the structure of compact dynamical systems, Funkcial. Ekvac., 13 (1970), 147-170.

10. P. Seibert and P. Tulley, On dynamical systems in the plane, Arch. Math., 18 (1967), 290-292.

11. T. Ura, Sur le Courant Extérieur à une Région Invariante; Prolongements d'une Caractéristique et l'order de Stabilité, Funkcial. Ekvac., Vol. 2, 1959, 143-200; nouv. edition 105-143.

Received November 10, 1970 and in revised form October 26, 1971.

OKLAhoma State UNIVERSity

AND

Northeast Missouri State College 



\section{PACIFIC JOURNAL OF MATHEMATICS}

\section{EDITORS}

\author{
H. SAMELSON \\ Stanford University \\ Stanford, California 94305

\section{R. HoвBY} \\ University of Washington \\ Seattle, Washington 98105
}

\section{J. DugundJI}

Department of Mathematics University of Southern California Los Angeles, California 90007

\author{
RichaRd ARENS \\ University of California \\ Los Angeles, California 90024
}

\section{ASSOCIATE EDITORS}
E. F. BECKENBACH
B. H. NEUMANN
F. WOLF
K. YoshidA

\section{SUPPORTING INSTITUTIONS}

\author{
UNIVERSITY OF BRITISH COLUMBIA \\ CALIFORNIA INSTITUTE OF TECHNOLOGY \\ UNIVERSITY OF CALIFORNIA \\ MONTANA STATE UNIVERSITY \\ UNIVERSITY OF NEVADA \\ NEW MEXICO STATE UNIVERSITY \\ OREGON STATE UNIVERSITY \\ UNIVERSITY OF OREGON \\ OSAKA UNIVERSITY
}

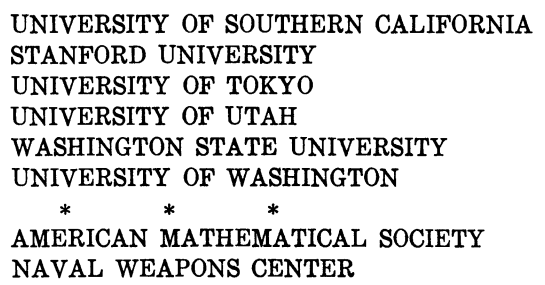

The Supporting Institutions listed above contribute to the cost of publication of this Journal, but they are not owners or publishers and have no responsibility for its content or policies.

Mathematical papers intended for publication in the Pacific Journal of Mathematics should be in typed form or offset-reproduced, (not dittoed), double spaced with large margins. Underline Greek letters in red, German in green, and script in blue. The first paragraph or two must be capable of being used separately as a synopsis of the entire paper. The editorial "we" must not be used in the synopsis, and items of the bibliography should not be cited there unless absolutely necessary, in which case they must be identified by author and Journal, rather than by item number. Manuscripts, in dup icate if possible, may be sent to any one of the four editors. Please classify according to the scheme of Math. Rev. Index to Vol. 39. All other communications to the editors should be addressed to the managing editor, Richard Arens, University of California, Los Angeles, California, 90024.

50 reprints are provided free for each article; additional copies may be obtained at cost in multiples of 50 .

The Pacific Journal of Mathematics is published monthly. Effective with Volume 16 the price per volume (3 numbers) is $\$ 8.00$; single issues, $\$ 3.00$. Special price for current issues to individual faculty members of supporting institutions and to individual members of the American Mathematical Society: $\$ 4.00$ per volume; single issues $\$ 1.50$. Back numbers are available.

Subscriptions, orders for back numbers, and changes of address should be sent to Pacific Journal of Mathematics, 103 Highland Boulevard, Berkeley, California, 94708.

PUBLISHED BY PACIFIC JOURNAL OF MATHEMATICS, A NON-PROFIT CORPORATION

Printed at Kokusai Bunken Insatsusha (International Academic Printing Co., Ltd.), 270, 3-chome Totsuka-cho, Shinjuku-ku, Tokyo 160, Japan. 


\section{Pacific Journal of Mathematics}

\section{Vol. 41, No. 2 December, 1972}

Tom M. (Mike) Apostol, Arithmetical properties of generalized Ramanujan sums .......................................... 281

David Lee Armacost and William Louis Armacost, On p-thetic groups ........ 295

Janet E. Mills, Regular semigroups which are extensions of groups .......... 303

Gregory Frank Bachelis, Homomorphisms of Banach algebras with minimal ideals ................................................ 307

John Allen Beachy, A generalization of injectivity .................. 313

David Geoffrey Cantor, On arithmetic properties of the Taylor series of rational functions. II.........................................

Václáv Chvátal and Frank Harary, Generalized Ramsey theory for graphs. III.

Small off-diagonal numbers .................................. 335

Frank Rimi DeMeyer, Irreducible characters and solvability of finite groups . . . . 347

Robert P. Dickinson, On right zero unions of commutative semigroups........ 355

John Dustin Donald, Non-openness and non-equidimensionality in algebraic

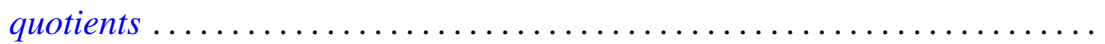

John D. Donaldson and Qazi Ibadur Rahman, Inequalities for polynomials with a prescribed zero ........................................ 375

Robert E. Hall, The translational hull of an $N$-semigroup ................ 379

John P. Holmes, Differentiable power-associative groupoids.............. 391

Steven Kenyon Ingram, Continuous dependence on parameters and boundary data for nonlinear two-point boundary value problems .

Robert Clarke James, Super-reflexive spaces with bases ..........

Gary Douglas Jones, The embedding of homeomorphisms of the plane in

continuous flows...............................

Mary Joel Jordan, Period $H$-semigroups and $t$-semisimple periodic

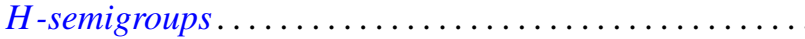

Ronald Allen Knight, Dynamical systems of characteristic 0

Kwangil Koh, On a representation of a strongly harmonic ring by sheaves...

Hui-Hsiung Kuo, Stochastic integrals in abstract Wiener space. ..

Thomas Graham McLaughlin, Supersimple sets and the problem of extending a

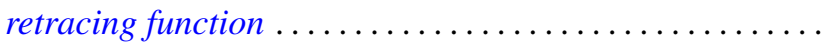

William Nathan, Open mappings on 2-manifolds .

M. J. O'Malley, Isomorphic power series rings

Sean B. O'Reilly, Completely adequate neighborhood systems and metrization

Qazi Ibadur Rahman, On the zeros of a polynomial and its derivative...

Russell Daniel Rupp, Jr., The Weierstrass excess function ..

Hugo Teufel, A note on second order differential inequalities and functional

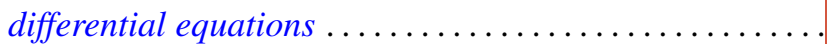

M. J. Wicks, A general solution of binary homogeneous equations over free 